\title{
Dermatological patients in the Intensive Care Unit - a hospital record based retrospective study
}

\section{Heena Gupta', Mrinal Gupta ${ }^{2}$}

${ }^{1}$ Department of Anaesthesiology, Government Medical College, Jammu, India, ${ }^{2}$ Consultant Dermatologist, Treatwell Skin

Centre, Jammu, India

Corresponding author: Mrinal Gupta, MD, E-mail: drmrinalgupta@yahoo.com

\begin{abstract}
Background: Various dermatological conditions can be life threatening and may require admission in the intensive care unit for monitoring and management. We carried out this study to assess the profile of dermatological admissions in the intensive care unit. Materials and Methods: It was a cross sectional retrospective study in which the records of all patients of various dermatological disorders admitted in the intensive care unit over a period of three years was studied and the clinical details were assessed. Results: Over a period of three years, a total of 16 patients (M:F 10:6), aged between $16-71$ years (mean $46 \pm 2.31$ years) were admitted with an underlying dermatological disorder. The most common condition requiring admission was Stevens Johnsons Syndrome $(n=5)$, followed by Pemphigus vulgaris $(n=4)$, erythroderma( $\mathrm{n}=2)$, pustular psoriasis, dermatomyositis, necrotizing faciitis, dapsone hypersensitivity syndrome and cutaneous lymphoma in 1 each. Interventions like mechanical ventilation were required in 2 patients, inotropic support in 2 patients, central venous line placement, renal replacement therapy and tracheostomy in one patient each. The mean duration of ICU stay was 14.2 days and mortality was the outcome in 2 patients of pemphigus vulgaris which was attributed to septicemia and shock. Conclusions: Dermatological disorders can cause significant morbidity and may require proper care in the intensive care unit. Timely initiation of intensive care can help in reducing the morbidity and preventing mortality.
\end{abstract}

Key words: Intensive care unit; Stevens Johnsons syndrome; Pemphigus vulgaris; Dermatological disorders

\section{INTRODUCTION}

Dermatological conditions are usually not considered life threatening by the general population and other medical specialties also, but certain skin conditions can cause significant morbidity and require specialized care and monitoring in the intensive care unit (ICU). Dermatological conditions like Stevens Johnsons syndrome, pemphigus vulgaris, erythroderma can cause acute skin failure which can result in temperature dysregulation, electrolyte imbalance and can predispose to secondary infections. These conditions require specialized care in the ICU and can lead to mortality in the absence of proper monitoring and care $[1,2]$.
We carried out this study to assess the profile and outcomes of patients with dermatological disorders admitted in the ICU.

\section{MATERIALS AND METHODS}

It was a cross sectional retrospective study in which the records of all patients of various dermatological disorders admitted in the intensive care unit of Government Medical College, Jammu over a period of three years was studied and the clinical details were assessed. The details of hospital stay, the interventions required and the clinical outcomes of stay were noted. 


\section{RESULTS}

Over a period of three years, a total of 16 patients (M:F 10:6), aged between 16-71 years (mean $46 \pm 2.31$ years) were admitted with an underlying dermatological disorder. The most common condition requiring admission was Stevens Johnsons Syndrome $(n=5)$, followed by Pemphigus vulgaris $(n=4)$, erythroderma $(n=2)$, pustular psoriasis, dermatomyositis, necrotizing faciitis, dapsone hypersensitivity syndrome and cutaneous lymphoma in 1 each. Interventions like mechanical ventilation were required in 2 patients, inotropic support in 2 patients, central venous line placement, renal replacement therapy and tracheostomy in one patient each. The mean duration of ICU stay was 14.2 days and mortality was the outcome in 2 patients of pemphigus vulgaris, which was attributed to septicemia and shock.

\section{DISCUSSION}

Intensive care unit (ICU) is a specialized unit to manage critically ill patients. Various skin conditions require intensive care owing to their severity and extent. Skin conditions like erythroderma, pustular psoriasis, toxic epidermal necrolysis (TEN), severe immunobullous disorders, congenital bullous disorders cause damage to or loss of large areas of skin leading to loss of skin function termed as acute skin failure [3].

Loss of the epithelium leads to exudation of fluid, electrolytes and protein of similar concentration to plasma. These losses of fluid, electrolytes and protein can lead to hypotension and consequent renal failure [3]. Fluid replacement by a peripheral line inserted through uninvolved skin is important for maintain the fluid balance in these cases. Increased cardiac output because of increased blood flow through the skin has can cause left ventricular failure in elderly patients with pre-existing ischaemic heart disease. Close monitoring of urine output and vital signs is essential and when there is major fluid loss and the threat of renal failure, central and arterial lines may be needed [4]. Mild congestive cardiac failure associated with increased blood flow to the skin will respond to treatment of the skin condition itself. More severe cases should be treated with diuretics and venodilators. If arterial oxygen concentration reaches a dangerously low level, mechanical ventilation may be required [3-5].

Patients with extensive skin disease are unable to regulate their body temperature because of inability to sweat and impaired control of cutaneous blood flow. Their temperature should be measured with a low reading thermometer. The room temperature should be increased to prevent shivering and calorie loss and regular monitoring of temperature is imperative to prevent hypothermia [4]. Maintain of nutrition is another important aspect in these patients owing to protein and fluid loss from the skin and may require nasogastric feeding. Maintenance of proper protein intake and regular monitoring of blood glucose is important in critically ill patients $[4,6]$.

Infection is a major cause of death in dermatological patients admitted in the ICU. The patients are predisposed to infections owing to the loss of skin barrier and immunocompromised states. Regular monitoring of temperature, blood pressure and urine output may help in identifying septicemia. Regular swabs and bacterial cultures should be performed to identify the causative organisms [4].

Dermatological disorders can cause significant morbidity and may require proper care in the intensive care unit. Timely initiation of intensive care can help in reducing the morbidity and preventing mortality.

\section{Statement of Human and Animal Rights}

All the procedures followed were in accordance with the ethical standards of the responsible committee on human experimentation (institutional and national) and with the 2008 revision of the Declaration of Helsinki of 1975.

\section{Statement of Informed Consent}

Informed consent for participation in this study was obtained from all patients.

\section{REFERENCES}

1. Gupta H, Gupta M. Dermatological manifestations in the intensive care unit - A prospective study. Our Dermatol Online. 2018;9:390-2.

2. Uwe Wollina, Andreas Nowak: Dermatology in the Intensive Care Unit. Our Dermatol Online. 2012;3:298-303.

3. Levine JM. Skin failure: an emerging concept. Journal of the American Medical Directors Association. 2016;17:666-9.

4. Hassan I, Rather PA. Emergency dermatology and need of dermatological intensive care unit (DICU). J Pakistan Assoc Dermatol. 2016;23:71-82.

5. Cohen KE, Scanlon MC, Bemanian A, Schindler CA. Pediatric skin failure. Am J Crit Care. 2017;26:320-8.

6. Harr T, French LE. Toxic epidermal necrolysis and Stevens-Johnson syndrome. Orphanet J Rare Dis. 2010;5:39.

Copyright by Heena Gupta, et al. This is an open access article distributed under the terms of the Creative Commons Attribution License, which permits unrestricted use, distribution, and reproduction in any medium, provided the original author and source are credited.

Source of Support: Nil, Conflict of Interest: None declared. 